\section{Coleccionistas de objetos históricos, arqueológicos y de ciencias naturales en museos municipales de la provincia de Buenos Aires en la década de 1950}

\section{Collectors of historical, archeological, and natural science objects at municipal museums in Buenos Aires province, Argentina, during the 1950s}

PUPIO, M. A.: Coleccionistas de objetos históricos, arqueológicos y de ciencias naturales en museos municipales de la provincia de Buenos Aires en la década de 1950.

História, Ciências, Saúde-Manguinhos, v. 12 (suplemento), p. 205-29, 2005.

En este trabajo se explorarán algunos aspectos ligados al proceso de formación de las colecciones arqueológicas en el contexto de creación y expansión de los museos municipales en la provincia de Buenos Aires en la década de 1950. Las colecciones analizadas surgieron en el ámbito privado, recolectadas por coleccionistas que las cedieron para dar origen a estos museos. Al mismo tiempo que las colecciones se hacían públicas, los coleccionistas se convirtieron en sus responsables en el rol de directores de las nuevas instituciones.

En el contexto de estos museos, los coleccionistas establecieron relaciones institucionales que sirvieron para elaborar estrategias comunes para el ingreso, selección y exhibición de las colecciones arqueológicas, conformando redes solidarias, como la descripta aquí, para el sur de la provincia de Buenos Aires.

PALABRAS CLAVES: Argentina, museos, coleccionistas, colecciones históricas, arqueológicas y de ciencias naturales.

PUPIO, M. A.: Collectors of historical, archeological, and natural science objects at municipal museums in Buenos Aires province, Argentina, during the 1950s. História, Ciências, Saúde-Manguinhos, v. 12 (supplement), p. 205-29, 2005.

Through reference to the creation and expansion of municipal museums in the province of Buenos Aires during the 1950s, the article explores some aspects of how archeological collections are compiled. The collections under study came from private hands, having been gathered by collectors who relinquished them so these museums could be formed. At the same time that these collections became public, the collectors themselves became responsible for them in the role of directors of the new institutes. Within this context, the collectors established institutional relations that allowed them to devise common strategies concerning the receipt, selection, and exhibition of archeological collections. The result was the shaping of a network of solidarity in the southern part of Buenos Aires province.

KEYWORDS: Argentina, museum, collectors, historical collections, archeological collections, natural science collections. 
Un loco el inglés, vino a hacer un Museo en este pueblo perdido, en medio de la indiferencia general. A nadie le interesa el pasado aquí, todos vivimos el presente. Si todo sigue igual desde siempre, para qué guardar los restos de lo que no ha cambiado.

(Ricardo Piglia, La ciudad ausente. Barcelona, Seix Barral, 1997, p. 110).
Este trabajo pudo realizarse gracias a un Subsidio de Inicio de Carrera de la Fundación Antorchas. Una versión del mismo fue presentada en 1998, en Río de Janeiro, en el V Congresso LatinoAmericano de História das Ciências e da Tecnologia, Gênero, Ciência e Tecnologia na História LatinoAmericana. Los errores son de mi autoría.

\section{Introducción}

L a década de 1950 presenció la creación de una serie de museos en el ámbito de los municipios de la provincia de Buenos Aires. Estas instituciones que surgieron con el auspicio privado o estatal podían contener colecciones históricas, de ciencias naturales, de bellas artes o temáticas (por ejemplo biográficas). En este trabajo se analizarán los museos históricos y de ciencias naturales, ya que en el período estudiado bajo estas denominaciones se encontraban instituciones que poseían fondos museográficos de carácter histórico, arqueológico, etnográfico, folklórico y de ciencias naturales.

La creación de museos, aunque no era un fenómeno nuevo, adquiría en esta década un relieve y características distintivas. En efecto, desde que en 1872 se fundó el primer museo municipal hasta fines de la década de 1940, abrieron sus puertas diecisiete museos municipales de historia y ciencias naturales, diez privados y siete municipales, mientras que sólo en los diez años siguientes lo hicieron nueve estatales y seis privados (Ministerio de Educación, 1979).

El surgimiento y expansión de estas instituciones estuvo íntimamente ligado al coleccionismo, y éstas tuvieron su origen en el traspaso de las colecciones del ámbito estrictamente privado al público. ${ }^{1}$ Este proceso de cesión dio origen a instituciones públicas que tenían por objetivo la protección, el estudio de las colecciones y la puesta en escena en un espacio diseñado para este propósito con el objetivo de estar disponibles para la comunidad de origen.

En Argentina, la creación sistemática de museos se remonta a la segunda mitad del siglo XIX, fenómeno que se consolidó hacia fines de siglo, momento en el que estaban funcionando varios museos científicos bajo la administración de los estados nacional y provincial. ${ }^{2}$ Las colecciones de estos centros fueron reunidas para ser estudiadas en los gabinetes científicos y ser exhibidas con el objetivo de educar al público en la historia, el arte y la ciencia. Estas instituciones, junto con otros centros científicos, universitarios y educativos actuaron como instituciones culturales relevantes en la creación de una identidad y una cultura nacional argentinas.

A diferencia de los museos metropolitanos del siglo XIX, a mediados del siglo $X X$, surgieron museos municipales en pequeñas 
ciudades de provincia por iniciativa de coleccionistas que recolectaban una amplia gama de objetos entre los que se encontraban los históricos, arqueológicos y de ciencias naturales y que hacen pensar en los antiguos gabinetes de rarezas (Podgorny, 2000a). Estas colecciones, que nacieron en el ámbito privado, fueron cedidas por los coleccionistas y dieron origen a estas instituciones. Estos museos emergieron en el ámbito municipal como un tipo especial de institución que tenían como principal objetivo arbitrar los medios para resguardar esos materiales, reconocidos de interés público, primero por los coleccionistas, luego por la comunidad y en algunos casos por autoridades municipales y/o provinciales. Todos estos museos municipales, privados o estatales pasaron a regirse por las mismas reglas - aunque no siempre escritas - de protección y exposición. Unos y otros tuvieron su origen y desarrollo en una sola persona, fenómeno que podríamos señalar como el museo de padre único. ${ }^{3}$ Según Farró Fonalleras (1995), aunque estos museos se caracterizaban por su sorprendente individualidad y la visión idiosincrática de su fundador, existen tendencias comunes que reclaman para ellos el reconocimiento de una tipología museográfica específica. Estos museos municipales poseían características similares no sólo porque tuvieron su origen en la persona del coleccionista, sino también por compartir un patrón común en el proceso de selección, interpretación y exhibición de los objetos, intensificado por los lazos de relación que establecieron entre ellos.

En este trabajo se explorarán cuatro aspectos ligados al proceso de formación de las colecciones arqueológicas. Por un lado, el perfil de los coleccionistas, el rol de éstos en el proceso de creación y expansión de los museos locales en este período y el establecimiento de redes de relaciones en el contexto de institucionalización. Por otro lado, en este trabajo se explorarán también los criterios de selección de materiales que aplicaron y las estrategias de obtención de los mismos. Para esto se ha trabajado con la correspondencia privada y pública de un grupo de siete coleccionistas, así como con las colecciones reunidas por ellos.

El análisis de la correspondencia privada y pública permitió detectar una red de relaciones entre coleccionistas en el sudoeste bonaerense facilitada por el proceso de institucionalización de las colecciones. La aquí señalada estuvo conformada por siete coleccionistas. Uno de ellos fue Antonio Crespi Valls, primer director del Museo y Archivo Histórico Municipal de Bahía Blanca (19511959). Luis Scalese y José Mayo estuvieron relacionados con la creación del Museo Histórico Regional de la Campaña al Desierto en 1943, luego Museo Histórico Coronel Villegas. Scalese ocupó el cargo de Director Honorario hasta su retiro en 1959, continuando José Mayo como Subdirector Honorario. El cuarto fue Gabriel 
Campomar Cervera, quien creó con su colección el Museo Histórico y de Ciencias Naturales de Salliqueló el 20 de julio de 1952 y fue su director hasta su muerte en 1975. Emma Nozzi fue Directora Honoraria del Museo Histórico Regional Francisco de Viedma desde 1951. Hugo Diez creó un museo privado en la ciudad de Laprida. Isaac Schasky fue un coleccionista de Rivera cuyos materiales fueron los únicos que no llegaron a conformar un museo.

Las ciudades mencionadas se encuentran ubicadas en la región sudoeste de la provincia de Buenos Aires, caracterizada en la década de 1950 por núcleos urbanos pequeños y grandes extensiones rurales debido a la intensa explotación agropastoril (Dirección General del Servicio Estadístico Nacional, 1951). De estos centros, Bahía Blanca fue el de mayor crecimiento y un nodo en las comunicaciones de la región, ya que fue sede de puertos de exportación de cereales y carne. Este lugar central en la región estaba dado por el sistema de comunicaciones vial (a través de rutas nacionales y provinciales) y la red ferroviaria que convergía a los puertos de la ciudad. Eso mantuvo a las localidades del sudoeste en permanente contacto con la ciudad de Bahía Blanca, ya que ofrecía servicios de salud, financieros, culturales y educativos (incluyendo la educación universitaria con la creación, primero, del Instituto Tecnológico del Sur en 1946, base de lo que posteriormente fue la Universidad Nacional del Sur, abierta en 1956) (Figura 1).

\section{Los coleccionistas}

Los coleccionistas mencionados en este trabajo eran autodidactas sin educación académica, cuya formación se basaba en lecturas aisladas y en la experiencia adquirida en el acto de coleccionar, entrenamiento que les había permitido seleccionar y organizar sus colecciones. Recordemos que la formación de museólogos en Argentina se impartía en dos instituciones universitarias. La Facultad de Filosofía y Letras de la Universidad de Buenos Aires había creado en 1923 el curso de Técnicos para el Servicio de Museos, dependiente de la carrera de Historia. En el año 1948, este curso se transformó en carrera independiente de dos años otorgando un diploma a los estudiantes y en 1950 el Consejo Superior de la Universidad realizó las modificaciones necesarias para darle una duración de tres años con quince materias, alcanzando en 1957 el número de veinte materias y conformando de este modo la carrera de Técnico para el Servicio de Museos que dependió del Departamento de Ciencias Antropológicas de la Facultad de Filosofía y Letras (Sanguinetti de Bórmida, 1963). La Universidad del Museo Social Argentino organizó la carrera de Auxiliares Técnicos con una duración de dos años que comenzó a funcionar en 1959, convertida luego en Licenciatura de cuatro años (Silva 


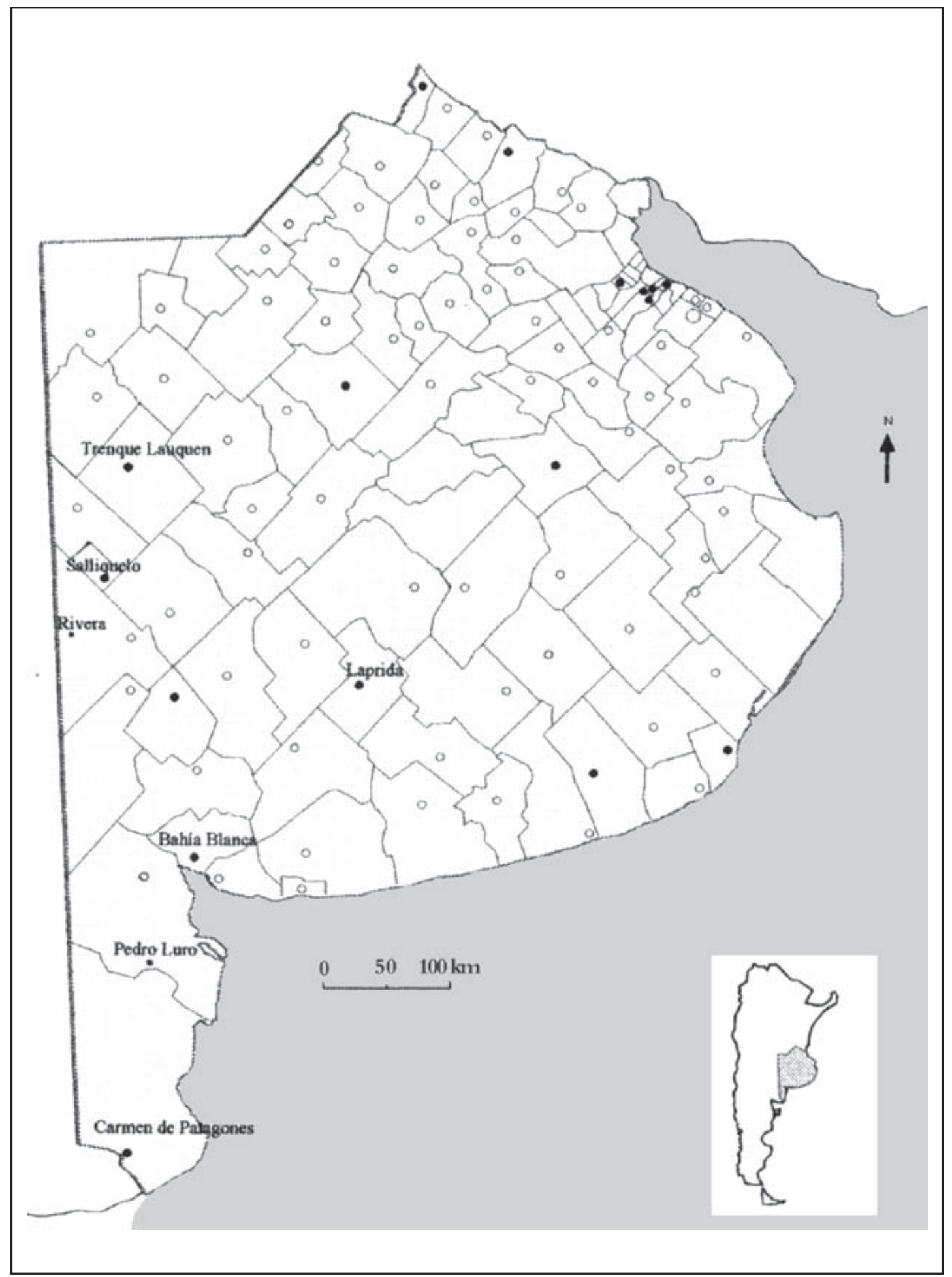

Figura 1: ubicación de los dieciseis museos locales (públicos y privados) abiertos en la década de 1950. Además se señalan las localidades de Trenque Lauquen cuyo museo pasó a depender de la adminstración provincial en 1951, Pedro Luro, sede del Museo de la Patagonia de la Orden Salesiana y Rivera, ciudad donde residia Isaac Schatzky. 
Montaner, 1959a, 1959b). De todos modos, cabe aclarar que ninguno de estos coleccionistas de ciudades de provincia optó por la formación profesional ofrecida por estas instituciones.

Sin embargo, en la comunidad local todos ocupaban lugares destacados, ya sea como docentes (Emma Nozzi), periodistas (Antonio Crespi Valls, Gabriel Campomar Cervera y Hugo Diez), farmacéuticos (José Mayo e Isaac Schatsky) o agente consular (Luis Scalese). Por la dedicación a la historia de la ciudad adquirían en los límites locales un prestigio equivalente al de 'historiador', 'arqueólogo' o 'escritor'. Este prestigio se consolidó ya que todos ellos produjeron un gran volumen de artículos en periódicos y publicaciones financiadas con dinero propio o con fondos municipales

La mayoría de los coleccionistas comenzaron a recolectar los materiales arqueológicos en la década de 1940 como producto de viajes de campo. Emma Nozzi logró reunir los materiales desde su acción como docente, a través de la coordinación de un periódico escolar en el que se trataban los temas del pasado indígena regional. De este modo, los alumnos comenzaron a acercar material y datos de 'paraderos' indígenas. El resto de los coleccionistas lo hicieron en sus momentos de ocio, como hobby y como consideración de una obligación cívica (Figura 2).

El tiempo dedicado a la recolección, en todos los casos, generó un volumen importante de materiales, lo que le dio a cada conjunto el status de colección y paralelamente le confirió un gran respeto a los coleccionistas. Este prestigio permitió que, junto con la cesión de las colecciones, la mayoría haya pasado a conducir estos museos. De esta manera, sus colecciones se hicieron públicas, convirtiéndose en objeto de protección y exposición en un espacio particularmente constituido para este propósito. Del mismo modo, los coleccionistas continuaron con la acción de coleccionar, ahora no ya en el ámbito estrictamente privado, sino en el público. ${ }^{4}$

Aun desde el rol de directores de museos, los coleccionistas como en el caso de Luis Scalese - se veían a sí mismos como 'diletantes' que realizaban un hobby de viejo coleccionista como producto de una formación autodidacta - en su caso en 'filosofía y letras'.${ }^{5}$ Veremos que en este grupo, con excepción de Crespi Valls, los coleccionistas le dan a su actividad un valor no profesional sino vocacional y de servicio.

En este grupo Isaac Schatsky fue el único que no cedió su colección para la apertura de un museo en la ciudad local. Por el contrario, el 26 de marzo de 1956 hizo la donación formal de su colección arqueológica, sus manuscritos inéditos y su biblioteca especializada a la cátedra de Etnografía del Departamento de Humanidades de la Universidad Nacional del Sur. Las autoridades universitarias mostraron interés en organizar el museo sólo en 1959. 
Figura 2

Coleccionista
Antonio Crespi Valls
Nació en Palmas de Mallorca
el 16 de enero de 1892.
Casado con Elisa Dominga
Anselmi el 3 de febrero de
1930, sin hijos.
Argentino nacionalizado.
Murió el 4 de febrero de
1959.

\section{Gabriel Campomar \\ Cervera}

Nació en Palmas de Mallorca en 1897.

A los 5 años se trasladó con su familia a la Argentina. Argentino nacionalizado. Soltero.

Murió en 1975.

\section{Formación}

Realizó sus estudios en Argentina, completando la escuela secundaria.

Terminó segundo
grado de la escuela
primaria en Argentina.

De pequeño ingresó a trabajar en una imprenta. primaria en Argentina. Comenzó a escribir y a editar revistas y libros de poesías. Entre 1938 a 1948 edita el semanario Nueva Era y a partir de 1950 el semanario Noticias. Director Honorario del Museo Histórico de Salliqueló.

\begin{tabular}{|c|c|c|}
\hline $\begin{array}{l}\text { Hugo Diez } \\
\text { Nació en } 1917 .\end{array}$ & $\begin{array}{l}\text { Maestro Normal } \\
\text { Nacional. }\end{array}$ & $\begin{array}{l}\text { Maestro. } \\
\text { Periodista, dueño del Diario } \\
\text { La Opinión. }\end{array}$ \\
\hline $\begin{array}{l}\text { Emma Nozzi } \\
\text { Nació en Guardia Mitre, Pcia. } \\
\text { de Río Negro el } 1 \text { de } \\
\text { noviembre de } 1917 .\end{array}$ & $\begin{array}{l}\text { Maestra Normal } \\
\text { Nacional. }\end{array}$ & $\begin{array}{l}\text { Maestra. } \\
\text { Directora Honoraria del } \\
\text { Museo Histórico Regional } \\
\text { Francisco de Viedma. }\end{array}$ \\
\hline
\end{tabular}

Murió el 27 de diciembre de 1999.

\section{Luis Scalese}

Nació en Italia el 6 de noviembre de 1882 Llegó a Argentina casado y con dos hijos (enviudó en 1912).
Terminó la escuela elemental en Italia.
Hasta 1910 trabajó en el campo. Ya en 1907 comenzó a escribir artículos literarios en periódicos locales.

Fue Agente Consular de Italia (1920 a 1943).

Trabajó en la Sociedad Italiana de Trenque Lauquen (1910-1939). Agente y corresponsal del diario La Nación (1943)

\section{Farmacéutico}

Sin datos. 
Al año siguiente, el centro de estudios contrató al Dr. Marcelo Bórmida para la organización de la asignatura Prehistoria General y para orientar la formación de un Museo Arqueológico y Antropológico que dependiera de esa universidad. ${ }^{6}$ Pero ya era tarde, la Colección Schatsky se hallaba en condiciones inadecuadas, los manuscritos no se encontraban y el contenido de las cajas estaba 'un tanto revuelto' en el subsuelo del actual edificio del Rectorado. ${ }^{7}$

Estos coleccionistas también eran reconocidos como amateurs por los funcionarios de las instancias institucionales superiores. Tomás Bernard, quien fue Director de Museos de la Provincia de Buenos Aires (1953-1955), describió este proceso de creación de museos como un 'amateurismo' (Bernard, 1957) propio de todos los comienzos:

Nuestros museos históricos locales y regionales, nacieron en base a colecciones reunidas por aficionados e investigadores, en algunos casos utilizando lo ya existente, en forma harto precaria. Vivieron del desinterés de unos pocos propulsores y de la devoción de unos cuantos patriotas, que son los auténticos salvadores de nuestro acervo patrimonial en lo histórico. Así se cumplió con sacrificio y cariño la dura etapa de la recolección del material y su preservación de las mil fuerzas destructoras y dispersoras. Los museos locales surgieron tras una férrea voluntad, aquí y allá, sin plan ni método, haciendo su acervo como podían, con material dispar y heterogéneo, las más de las veces en absoluto divorcio con las normas técnicas y los dictados de la ciencia... (Bernard, 1957, p. IV-V.)

Tal como se observa en este relato, el 'amateurismo' era entendido como un estado embrionario que necesariamente debía ser superado para alcanzar la profesionalización que los museos requerían en ese momento.

\section{Estrategias de institucionalización de los museos}

En la década de 1950 en el ámbito de la provincia de Buenos Aires, se produjo un cambio en la organización administrativa de los museos. Durante los gobiernos peronistas de Domingo Mercante (1946-1951) y Carlos Vicente Aloe (1951-1955) se promovió la creación de museos y una política centralizada en su manejo, ya que se los consideraba como instituciones pedagógicas cuyas acciones, al igual que la educación, debían ser minuciosamente planificadas en el contexto de una política cultural del estado.

La reforma constitucional provincial de 1949 creó el nuevo diseño administrativo en el que se incluía el Ministerio de Educación, cambiando la estructura institucional a cargo de la gestión educativa y cultural. Con la creación del Ministerio de Educación en 1949 su 
acción quedó dividida en tres subsecretarías: a) Administrativa, b) Educación y c) Cultura. En el marco de la Subsecretaría de Cultura fue creada la Dirección de Museos Históricos, centralizando bajo su administración los cuatro museos provinciales ya existentes y que antes dependían de organismos diferentes: Colonial e Histórico de Luján (1917), Gauchesco Ricardo Guiraldes de San Antonio de Areco (1938), Evocativo de Dolores (1939) y el Pampeano de Chascomús (1941). A estas cuatro instituciones se agregaron después de 1950 los museos de la Reconquista en Tigre, Museo y Archivo Dardo Rocha en La Plata, Museos Regionales Almirante Guillermo Brown en Quilmes y General Conrado Villegas en Trenque Lauquen y en 1953 el Museo y Biblioteca de Lobos. Esta estructura administrativa se completó con la organización del Ministerio de Educación en 1953 en la que la Dirección de Museos se convirtió en Dirección de Museos y Reservas Históricas y poco tiempo después en Dirección de Museos, Reservas e Investigaciones Culturales, ampliándose de este modo el ámbito de acción específica de la dirección.

La acción de estos organismos tuvo por objetivo la centralización de la administración y el control de la política cultural en el ámbito de la provincia. En este contexto se dictó el primer 'Reglamento General para los museos históricos provinciales' en 1953 que fijaba la estructura interna de los mismos, su coordinación funcional y los principales aspectos técnicos, culturales y administrativos. ${ }^{8}$ Con esta acción, la Dirección tenía como política elaborar una propuesta integral para que todas las instituciones museísticas del territorio de la provincia, dependientes del poder provincial, municipal o de la órbita privada se rigieran bajo pautas comunes. En este sentido, en el Congreso Extraordinario de Municipalidades reunido en la ciudad de la Plata, en 1954, se propició la coordinación con las municipalidades para que éstas uniformen y armonicen su régimen técnico y de organización de los museos locales o regionales bajo su dependencia sobre la base del sistema establecido para los museos históricos provinciales por el Ministerio de Educación (Ministerio de Gobierno, 1954, p. 109-10). Del mismo modo, en 1954 se coordinó un programa de auxilio a los museos y colecciones particulares de interés público (Anales de Legislación Argentina, 1954, p. 1784).

La acción centralizadora de la Dirección tuvo su punto máximo en julio de 1955 al diseñarse una política por la cual cada museo provincial tenía un área de influencia y a cargo un grupo de museos municipales a los que debía controlar y supervisar, dictando las áreas temáticas que cada institución debía desarrollar. Este proyecto tenía como objetivo dar unidad orgánica e integración sistemática a los servicios que debían brindar los museos oficiales en el territorio bonaerense. ${ }^{9}$ Este proyecto no se llevó a cabo debido al golpe militar de septiembre de 1955 que produjo un cambio ideológico y de gestión importante. 
En este contexto institucional, surgido a partir de 1950, los coleccionistas eligieron distintas estrategias para hacer públicas sus colecciones, siempre cuidando sostener su liderazgo en esa decisión. Los coleccionistas consideraban que el presupuesto personal e institucional con el que contarían para darle continuidad a la institución dependería de la maniobra elegida para hacer públicas las colecciones. De este modo, mientras algunos museos surgieron y se mantuvieron en jurisdicción municipal (Museo Histórico y de Ciencias Naturales de Bahía Blanca), otros continuaron en la órbita particular (Museo Histórico La Opinión de Laprida) y el resto intentó pasar al ámbito provincial para asegurar la continuidad de la institución. Esta última decisión estuvo fundada en la confianza que generaba la Dirección de Museos.

El museo de Bahía Blanca fue creado como Museo y Archivo Histórico Municipal en 1943 dentro de la estructura administrativa municipal. Esta institución, que surgió con las secciones de arqueología, historia, etnografía, antropología y cartografía, abrió al público el 29 de octubre de 1951 como Museo Histórico y en 1955 pasó a denominarse Museo Histórico y de Ciencias Naturales debido a la relevancia que cobraron las colecciones paleontológica, mineralógica, botánica y zoológica (Arecco, 1955). Antonio Crespi Valls fue nombrado conservador de la institución creada en 1943 y luego director en 1951, cargo que ocupó hasta su muerte el 4 de febrero de 1959. Tanto en el caso de la creación como de la apertura al público, se manifestaba la necesidad de contar con una institución de estas características como política de estado municipal. Tal como señaló Antonio Crespi Valls en el discurso inaugural del Museo, estas instituciones debían ser protegidas por la comunidad en su conjunto:

Toca a todos nosotros engrandecerlos y cuidarlos. Nadie puede desentenderse de hacerlo por despreocupación o por ignorancia. La historia atañe por igual a quienes ya fueron y a los que ahora somos. Aquel que niega la historia se niega a sí mismo. Nosotros no somos más que los continuadores del ayer, que estamos trabajando hoy, esperando que el mañana nos convierta, también en Historia (Boletín Municipal, 1951, p. 12.084).

El Museo Histórico Regional de la Conquista del Desierto de Trenque Lauquen surgió en abril de 1943 como propiedad del Instituto Cultural de esa ciudad. A pocos años de su creación, funcionó en la casa particular de Luis Scalese, quien fue hasta su retiro, en agosto de 1959, su Director Honorario. Las dificultades económicas y edilicias, y la ausencia de voluntad del municipio por hacerse cargo del museo, llevaron a la decisión de efectuar la donación de la colección a la provincia. Fundamentó la necesidad de traspaso, a través del uso de un discurso en el que siempre se 
colocaba como víctima del poder estatal. En principio, las quejas se dirigieron a las autoridades municipales, a quienes le reclamaba el escaso apoyo de $\$ 20$ moneda/nacional mensuales de subvención y no pagarle una renta por la casa donde funcionaba el museo y en la que él también vivía. Esto se manifiesta en casi toda la correspondencia mantenida con colegas y colaboradores. ${ }^{10} \mathrm{La}$ Dirección de Museos aceptó la donación por decreto provincial del 27 de octubre de 1951, incluyendo la continuidad de Scalese como Director Honorario, y cambió la denominación de la institución por la de Museo Histórico Regional General Conrado Villegas la que pasó a depender técnica, presupuestaria y administrativamente de esa dirección. A pesar de esta situación, el organismo provincial no proveyó al museo de edificio ni de cargos, incluso nunca pagó alquiler alguno a Scalese, propietario de la vivienda compuesta de "salón, saloncito y tres piezas con patio y cuartito en un terreno de 10 x 30 frente a la plaza de la ciudad" donde seguiría funcionando la institución hasta 1963. Los reproches iniciales se hicieron luego extensivos a los funcionarios provinciales, a quienes solicitaba el pago de alquiler ya que era su única propiedad y no tenía jubilación. Además, consideraba que este era un reclamo justo porque su vivienda tenía un cierto valor inmobiliario, dado que la casa lindante se había vendido en 1952 en \$ 200.000 m/n. A pesar de los insistentes pedidos, la Dirección de Museos solo producía erogaciones referidas a cuestiones administrativas mínimas como artículos de librería y la concesión de un cargo de portero de escuela para las tareas de limpieza. ${ }^{11}$ Nuevamente la correspondencia fue la arena en la que repetidamente Scalese manifestaba a sus pares el estado de abandono y desatención agudizado, según él, por la distancia entre el discurso político que declamaba un interés y preocupación que no se relacionaba con la realidad:

Verdaderamente son cosas nuestras, sin arraigo en los dirigentes políticos que miran sus conveniencias y dejan a nosotros los ilusos a seguir una obra, que efectivamente la hacemos como un HOBY de viejos. ¡Ya vendrán tiempos mejores! ${ }^{12}$

Con el mismo criterio, Gabriel Campomar Cervera consideraba que la única forma de asegurarle continuidad a su institución era consiguiendo el apoyo de la provincia, y luego de muchos años de quejas por la desidia y el desinterés del municipio y de la comunidad decidió, en 1965, donar las colecciones arqueológicas e históricas que componían el Museo Histórico y de Ciencias Naturales de Salliqueló para organizar en la ciudad un Museo Regional de jurisdicción provincial. Solicitó retener la dirección del mismo, esto último debido a que consideraba que: 
Me induce hacer esa insinuación el deseo de seguir colaborando en una obra que ha costado ingentes sacrificios, personales $y$ materiales, ya que no creo sea redundante hacer resaltar el esfuerzo que significa reunir los centenares de ejemplares líticos, recogidos en médanos de la zona y que demanda tareas arduas durante muchos años, realizadas con el solo propósito de hacer obra cultural de bien común. Solamente un conocedor de la zona puede suponer lo que significan esas tareas efectuadas en médanos enormes, en busca de paraderos indígenas y en todas épocas. ${ }^{13}$

Es importante señalar que toma esta decisión luego de consultar, entre otras personas, a Luis Scalese, quien le informa no sólo acerca de los pasos administrativos que debía seguir, sino de lo desgastante que era la realización de esos trámites, ejemplificándolo con su propia historia (la no adjudicación de presupuesto, el no pago de alquiler de la vivienda, la provisión de un único empleado, todo esto para un "museo de 8.167 piezas con un valor simbólico de $\left.\$ 24.393 \mathrm{~m} / \mathrm{n}^{\prime \prime}\right) .^{14}$

El Museo Histórico Municipal Francisco de Viedma también quiso contar con el apoyo de la provincia pero el Intendente Municipal de Carmen de Patagones ofreció al Ministerio de Gobierno la 'cesión de la tutela, dirección y administración', no su donación, reservándose además el derecho de proponer la constitución de los miembros que integrarían la Comisión "Amigos del Museo". Estas condiciones no fueron aceptadas por la provincia, quedando en jurisdicción municipal. ${ }^{15}$ Es interesante observar las consideraciones que elaboró Antonio Crespi Valls ante la consulta de Emma Nozzi, quien ocupaba el cargo de Directora Honoraria del museo. Él defendía el carácter estatal de los museos, en especial dentro de la órbita municipal, lo que aseguraría - a su entender - su funcionamiento por regirse bajo las reglas de la administración pública. Esto suponía contar con presupuesto municipal, provisión de empleados, horarios de atención al público regulados y edificios, que aunque no fueran construidos para tal fin y contaran con algunas deficiencias, permitían cumplir su función. ${ }^{16}$ En una carta en respuesta al consejo solicitado por la directora, estableció el por qué de su defensa en contra de las opciones de una administración privada o provincial, entre las que se debatía el Museo de Carmen de Patagones:

En todas las iniciativas de carácter, la buena voluntad particular alcanza hasta cierto límite de desarrollo; fuera de éste o sobrepasado, los propósitos mejor concebidos pueden fracasar por falta de organización, conocimientos o dificultades económicas, lo cual significa que debe pasar de la órbita privada a la oficial o a la de alguna institución que pueda contar con los 
recursos necesarios. A mi criterio, estos Museos deben ser muy, excesivamente, celosos de su autonomía, es decir, extremadamente localistas, para evitar caer en un centralismo desmoralizador que acaba por invalidar las más entusiastas iniciativas. Espero que usted comprenda la idea expuesta.

En el caso que usted expone, respecto al Museo de Patagones, quizá empezaron mal: Sólo hay dos clases de Museos: el oficial, en el caso creado por Ordenanza Municipal y el privado, pero éste no puede subsistir por mucho tiempo, si se hace cuestión de política, de creencias o ideologías en vez de hacer puramente historia. Cuando se quiere conformar a todo el mundo se acaba por descontentar a todos. ${ }^{17}$

La decisión de donar las colecciones a la provincia, a pesar de saber que no era la solución óptima, respondía a una situación de abandono de las autoridades municipales. Pero también, y de acuerdo a lo expresado por Luis Scalese y Gabriel Campomar Cervera, debido a las características que imponía la topografía de la pampa bonaerense que convertía la actitud de los coleccionistas en un comportamiento singular que aunque los distinguía de sus conciudadanos, no alcanzaba para lograr el apoyo de la comunidad y/o de las autoridades municipales:

El drama es siempre de orden económico. Literatos sin fortuna material y la riqueza intelectual en ambientes de compra-venta de hacienda, es muy relativa. Este Museo ha pasado al Gobierno de la Provincia, es decir aceptó la donación después de un año y medio de trámite y ahora no sabemos cuanto tardará en la ayuda económica. ${ }^{18}$

Por aquí, como por otros lados, pues no creo que sea un patrimonio salliquense, sólo vacas y sólo trigo... La juventud... sólo mujeres y naipes, más naipes que mujeres...y ¡Viva la Pepa! ...Yo no puedo atender el Museo, se llena de polvo, hay que renovar letreros, hay que hacer limpieza, hay que ordenar, y todo ello, me enferma el no poder hacerlo; entonces ¡que se vaya! ...que lo lleven, que se ocupe otro. Pero ese 'otro' no aparece y sigue lo mismo que antes... ${ }^{19}$

Hugo Diez optó por mantener su colección como museo privado, haciéndose cargo de su sustento y de su apertura al público en los talleres de su periódico, La Opinión. Esta situación se mantuvo hasta la ubicación de su colección en un edificio adecuado para museo, el cual fue inaugurado el 10 de septiembre de 1987 como museo privado administrado por una comisión de vecinos.

Más allá de las soluciones administrativas que hayan elegido los coleccionistas, y las dificultades que enfrentaban para la continuidad de los museos, la apertura de los mismos, tal como 
habíamos sugerido, generó un espacio de comunicación entre estos coleccionistas/directores. La acción de la Dirección de Museos ayudó a fortalecer estas redes de relaciones a través de la organización de reuniones de trabajo. En enero de 1952, en los días 27, 28 y 29, se realizó, en Carmen de Patagones, el Primer Congreso Provincial de Museos Históricos y Regionales de la Provincia de Buenos Aires y Zona Patagónica. Este encuentro tenía como objetivo establecer normas técnicas uniformes en materia de organización de los museos (inventarios, ficheros, archivos, sistemas de exposición, iluminación, conservación y restauración de piezas, vigilancia y previsión de incendios y robos, incremento del acervo) así como "determinar un sistema de mutua y permanente cooperación e intercambio técnico y cultural" ${ }^{20}$ Este encuentro en el que participaron Luis Scalese (subvencionado por la Dirección de Museos Históricos de la Provincia al enviarlo como delegado), Antonio Crespi Valls (con viáticos de la Municipalidad de Bahía Blanca) y por supuesto Emma Nozzi, quien ofició de anfitriona, permitió afianzar aún más la relación entre ellos. Es importante destacar que la comunicación se mantuvo ajena al trato oficial entre instituciones, creando una "vinculación espiritual, superior a todas las comunicaciones oficiales y escritas, ya que deja un margen de recuerdos imborrables" ${ }^{21}$ Esto se manifiesta en el tono utilizado en la correspondencia que resulta intimista con el uso de encabezados como 'estimado amigo' y con referencia a sucesos de la vida privada o de problemas institucionales que no se relatarían a otros funcionarios. Por otro lado, es en este carácter amistoso de la relación donde los coleccionistas percibían que iban a obtener los mayores resultados para los museos que ellos dirigían, ya que les permitía establecer una relación no circunscripta a las normas administrativas. Esta convicción es manifestada en la correspondencia posterior al encuentro, como la emitida por Luis Scalese y Emma Nozzi:

(...) se me ocurre pensar que uno de sus resultados más promisorios es, sin duda, la relación afectiva establecida entre los señores Delegados, circunstancia que habrá de proporcionar en lo futuro innumerables ventajas de todo orden, espiritual y material, a los Directores de Museos Históricos y Directores de Archivo. ${ }^{22}$

Sin duda alguna, la relación amistosa - enraizada en el conocimiento personal y directo - entre los señores Delegados, será uno de los más serios resultados de la reciente Asamblea y el que habrá de brindar los más apreciables y significativos frutos..$^{23}$

Esta reunión se muestra como el punto de partida de una relación profesional y personal que duraría toda la década y entre otras 
acciones incluiría el pedido y ofrecimiento de consejos, el intercambio de piezas y bibliografía y la realización de salidas de campo compartidas. Otro espacio de intercambio de información y presentación de ponencias había sido el Primer Congreso de Historia de los Pueblos de la Provincia de Buenos Aires realizado en la ciudad de La Plata en 1951. Fue organizado por el Archivo Histórico de la Provincia de Buenos Aires, cuyo Director Honorario era Ricardo Levene, quien propiciaba el intercambio de trabajos con los autodidactas de las ciudades de la provincia y colaboraba asesorándolos en cuestiones relativas a la historia local. Participaron de este congreso José Mayo y Luis Scalese como delegados de la comuna de Trenque Lauquen, Isaac Schatzky, delegado de Adolfo Alsina y Antonio Crespi Valls, delegado de Bahía Blanca (Archivo Histórico de la Provincia de Buenos Aires, 1951).

La creación de estos museos generó un clima propicio para la conformación de redes de relaciones institucionales y personales entre coleccionistas con el objetivo de promover tanto la creación de nuevos museos como la continuidad de los ya establecidos, impulsar el ingreso permanente de materiales, así como asegurar su lugar en estos establecimientos.

\section{Mecanismos de selección y obtención de las colecciones arqueológicas desde los museos}

Los fondos museográficos de estas instituciones estaban integrados por colecciones arqueológicas, faunísticas, florísticas, mineralógicas y objetos históricos (entre ellos documentos, fotografías y vestimentas), estos últimos pertenecientes a los primeros pobladores de la localidad.

Este conjunto de objetos, aunque parece representar una miscelánea desordenada, muestra, por el contrario, una visión organizada del mundo físico y social externo. Como toda colección de museo, las aquí analizadas han sido recolectadas con algúngrado de intención, lo que transformó parte del mundo natural y social en un objeto y una pieza de museo. El concepto que integraba esta diversidad era lo regional, el territorio era la unidad que se encerraba en el museo, y todo lo tradicional que integraba ese territorio tenía lugar en esos edificios. Las colecciones reunidas tenían como objetivo ser la representación totalizadora de la región, exhibidas de acuerdo a un tipo de narrativa visual expresada en secuencias de objetos que mostraban esta cosmovisión. De acuerdo a esto, los materiales a través su puesta en escena enseñaban la historia local, legitimando tanto un sistema de creencias como de prácticas profesionales - las de los coleccionistas.

Las colecciones históricas y arqueológicas, aunque relataban el pasado regional, poseían características distintivas que las colocaban 
en una esfera distinta de obtención y posesión. Las primeras pertenecían al dominio estrictamente local y representaban lo típico que cada museo poseía, cada objeto histórico remitía a un propietario, había pertenecido a algún ciudadano 'destacado' de la ciudad, y una vez ingresado al museo seguía manteniendo su relación de pertenencia: la biblioteca del primer médico, el instrumental de la primera partera. Eran en este sentido objetos únicos e irrepetibles y aunque ingresaran al museo en propiedad, la actitud que tomaba la institución era de custodia. Por otro lado, no eran objeto de intercambio y/u obsequio. En cambio, las colecciones arqueológicas pertenecían a una escala más amplia territorialmente que los límites de la ciudad y daban cuenta de un pasado regional cuyo sujeto histórico estaba caracterizado genéricamente como 'grupos indígenas de la región', sin considerar la dimensión temporal e histórica que este material representaba. Estos objetos sin 'dueños' pasaron a ser 'propiedad' del coleccionista, y en un gesto extremo de dominio sobre el conjunto de los objetos, Crespi Valls colocaba su sello (COLECCIÓN ANTONIO CRESPI VALLS) a todos los objetos recolectados por él, convirtiéndose en su dueño. Estas diferencias producían estrategias diferentes de obtención. Mientras los objetos históricos eran adquiridos mediante cesión de su propietario anterior, los arqueológicos eran poseídos a través de distintas estrategias. ${ }^{24}$

Las colecciones arqueológicas fundamentalmente tenían su origen en la recolección de campo. Cada uno de los coleccionistas poseía un conocimiento regional que los llevaba a recorrer sistemáticamente el área en busca de 'paraderos' indígenas, los cuales consideraban como puntos en el paisaje que contenían evidencias de vestigios indígenas. Gran parte de su actividad estaba orientada a la localización de estos paraderos para relevarlos, fotografiarlos, recolectar el material, inventariarlo y exhibirlo con el objetivo de realizar un mapa regional de las ocupaciones del área. En el campo, su actividad se centraba en la recolección superficial de objetos a los que se clasificaba en clases de artefactos formales. De este modo, estas colecciones estaban conformadas por un conjunto de piezas compuestas por material de molienda, puntas de proyectil, cerámica, raspadores y boleadoras. Cada uno de estos objetos era ordenado de acuerdo a su morfología, obteniendo de este modo subclases: boleadoras con y sin surco, puntas apedunculadas, con pedúnculo. El objetivo era siempre obtener una nueva tipología, no sobrecargar con piezas de una misma clase, tal como aconsejaba el mismo Crespi Valls:

me alegra muchísimo que sus andanzas por esos campos de arena les resulten fructíferas. Es los menos que el Destino puede darle a quienes sin temor al frío, a los vientos y al cansancio de las máquinas se lanzan a incursionar en el territorio de la 
arqueología, en el corazón de los médanos. Ahora bien, amigos, no vayan a caer en la tentación de convertir su hermoso Museo en un depósito. ¡Nada de esto! No vale un Museo por la cantidad de piezas guardadas y expuestas, sino por la calidad y variedad de ellas. Quiero decir, que si se tienen treinta pilones idénticos, hallados en el mismo paradero, nada supone el exhibir los treinta. Lo valioso es encontrar alguno distinto, ya en forma o material. Me explico. ${ }^{25}$

Los objetos repetidos, siempre presentes en las colecciones arqueológicas, permitieron dos nuevas estrategias de obtención una vez convertidas en piezas de museo: el obsequio y/o intercambio personal e institucional. ${ }^{26}$ Eran 'excedentes' que tenían la función de entrar en la esfera de intercambio, como establecía Crespi Valls: "aumenta nuestra colección y sobre todo proporciona la manera de retribuir algunas atenciones, con piezas muy repetidas". ${ }^{27}$

El obsequio y/o intercambio se producía en la esfera circunscripta al grupo de coleccionistas, entre ellos estaba permitido intercambiar objetos bajo la idea de que las piezas repetidas eran fuente de intercambio y regalo personal. La compra/venta de piezas no fue un mecanismo de intercambio utilizado, quedando como objetos protegidos contra la mercantilización. El único valor monetario que se les adjudicaba era un valor simbólico. ${ }^{28}$ En este sentido, eran objetos que atravesaron el proceso de singularización - en el sentido dado por Kopytoff (1991) - a través de una circulación restringida que los confinó a una esfera muy estrecha de intercambio cuyos términos y condiciones eran de conocimiento común en un grupo en el que las partes tenían categorías de percepción y valoración idénticas (Bourdieu, 1997).

Finalmente, debemos destacar otro mecanismo para ingreso de piezas al museo: la donación de particulares que incluía una lista de objetos de diversa naturaleza con el objetivo de ser exhibidos. En la correspondencia se observa el relato de las donaciones recibidas:

siempre recibo alguna donación de amigos que se hallan lejos, así he recibido flechas de Esquel, distintas a las de la zona, estoy esperando del Chaco, también he recibido una cajita con tierra de la Antártida; he recogido la mayoría de un esqueleto hallado en un médano, he conseguido un farol que usaba la policía nocturna de Nueva York... en fin, poco a poco se van juntando piezas de algún valor. ${ }^{29}$

Para que el sistema de donaciones funcionara permanentemente, Crespi Valls generó una red de donantes a través de la Comisión Honoraria "Amigos del Museo" del Museo Histórico. El sistema de donaciones ya estaba previsto en la ordenanza de creación del museo (6 de abril de 1943) que establecía un sistema de compensación a los 
donantes que prestaban colaboración. El Poder Ejecutivo les entregaría los siguientes diplomas: Miembros correspondientes, a los estudiosos residentes en el país o en el extranjero que se destacaran mediante publicaciones, estudios especiales o hubieran colaborado en la selección y ordenación de piezas y documentos de pertenencia del instituto; Miembros Honorarios, a los donantes de colecciones completas y de mucha importancia; Miembros Colaboradores, a los donantes de piezas y documentos sueltos (Municipalidad de Bahía Blanca, 1943). Cuando el museo abrió sus puertas, este sistema fue simplificado y así la Comisión de Amigos quedó integrada por Miembros de Número, residentes en la ciudad, y Miembros Corresponsales para los colaboradores radicados fuera de la ciudad. Isaac Schatzky fue designado, en 1951, Miembro Corresponsal del Museo por el Intendente Municipal Ing. Norberto Arecco, lo cual servía para ser presentado para facilitar las investigaciones arqueológicas.

De este modo, quedaba asegurado el ingreso permanente tanto de objetos como de información de paraderos arqueológicos y sitios paleontológicos. El mismo sistema estableció Gabriel Campomar Cervera quien contó con un grupo de vecinos que lo acompañaban permanentemente al campo: José Latasa, Julio Araco, Oscar Tuiz, Eduardo Paternessi y Víctor Mayo.

El funcionamiento de estas estrategias permitió que el acto de coleccionar en el ámbito de los museos no fuera ya un acto estrictamente individual, sino una acción compartida entre coleccionistas. Esto los colocó en una red solidaria de comunicación y cooperación basada en relaciones personales y profesionales y en la que todos los miembros poseían igual jerarquía, aunque reconociendo 'maestros'. En el grupo descripto, este rol era ocupado por Antonio Crespi Valls, quien actuó como asesor técnico en las salidas de campo y en los trabajos de procesamiento y exhibición del material. En particular su colaboración era requerida por Gabriel Campomar Cervera, tal como él mismo lo destacó en su semanario Noticias: “Nos ha visitado el Director del Museo Histórico de Bahía Blanca. Contestando a un pedido de asesoramiento del señor José Latasa y de nuestro director, el Intendente de Bahía Blanca, Ing. Don Norberto E. E. Arecco y el Secretario de Cultura y Asistencia Social, señor Arnaldo Collina, demostrando un alto espíritu de patriótica colaboración, y a la vez que su buena acción de gobernantes no se reduce a su zona sino que abarca el litoral" (Noticias, 1952).

En este caso, Crespi Valls había sido enviado por el Intendente de Bahía Blanca para salir al campo a localizar paraderos indígenas y recolectar materiales sobre la base de la necesidad del desarrollo de actividades culturales no sólo en la ciudad, sino en la región, en la cual Bahía Blanca ocuparía un lugar central. 
Del mismo modo, actuaba como consejero y así permanentemente sugería a Campomar Cervera que preparara gente que pudiera continuar con la obra del museo que tanto esfuerzo personal y monetario requería:

Su pasión por la Arqueología es profunda y sincera, y por lo tanto dedica a ello y su divulgación cuantos instantes puede, aun robándola a otras actividades más productivas. Pero su entusiasmo no puede ser compartido por otros con la misma intensidad. Muchas personas se apasionan un tiempo por una actividad espiritual cualquiera, pero poco a poco van 'aflojando', o dejando las cosas 'para mañana'. Lo esencial, en estos casos, es tener el tacto de formar continuamente 'nuevos discípulos' que ocupen el lugar de los que deserten o se vuelvan apáticos. ¿Usted me entiende? ${ }^{30}$

Estos coleccionistas consideraban necesaria la formación de discípulos, aunque no fuera una tarea fácil de lograr por los esfuerzos que implicaba, ya que estimaban que estas colecciones tenían una proyección para el conocimiento regional y para el disfrute de la comunidad. Por eso, en los trabajos de campo, contaban con un grupo de ayudantes, considerados como discípulos y a los cuales se debía enseñar el trabajo porque reconocían que su tarea no podía quedar limitada a la capacidad y esfuerzo individual.

\section{Consideraciones finales}

La correspondencia existente en los archivos administrativos de los museos de provincia ha brindado la posibilidad de estudiar algunos aspectos del proceso de formación de las colecciones arqueológicas en el contexto de creación de museos locales en la provincia de Buenos Aires a mediados del siglo XX.

Las colecciones arqueológicas, junto con las de ciencias naturales e históricas, tuvieron su origen en el ámbito estrictamente privado, recolectadas por coleccionistas/autodidactas, quienes utilizaban su tiempo libre y subsidiaban la tarea con otros empleos. En todos los casos, los coleccionistas manifestaron la necesidad de ceder sus colecciones para crear museos - tanto de propiedad privada como estatal - para poner en escena para la comunidad de origen un conjunto de bienes que daban cuenta del pasado geológico, arqueológico e histórico de la localidad. Al mismo tiempo que las colecciones se hacían públicas, los coleccionistas se convirtieron en sus responsables en el rol de directores de las nuevas instituciones como muestra del prestigio que les confería la colección, en prueba de su curiosidad, empeño y generosidad, ya que eran portadores de un conocimiento poco habitual, pero que servía al bien común. 
En el contexto de estos museos, los coleccionistas/directores establecieron relaciones institucionales, incentivadas por la política de gestión cultural implementada por el gobierno peronista de la provincia de Buenos Aires. De todos modos, este contacto tomó un carácter personal y en muchas ocasiones con posturas críticas hacia las autoridades municipales y provinciales. Estos vínculos sirvieron para elaborar estrategias comunes para el ingreso, selección y exhibición de las colecciones arqueológicas, conformando redes de relaciones solidarias como la descripta para el sudoeste de la provincia de Buenos Aires, caracterizada en general por la simetría entre sus participantes, aunque algunos de sus miembros hayan sido reconocidos en su rol de 'maestros'.

\section{CITAS}

${ }^{1}$ El coleccionismo es un fenómeno de extensa distribución espacial y temporal que tiene por finalidad dar origen a una 'colección'. Esta está conformada por un conjunto de semióforos (Pomian, 1990) reunidos por un coleccionista de acuerdo a un proceso de selección que le da sentido y unidad, recolectados con algún grado de intención por un particular que aleja a los objetos del propósito primario de su existencia y son mantenidos temporal o permanentemente fuera del circuito económico. El proceso de formación de colecciones ha sido estudiado desde muy variadas perspectivas, entre las que se encuentra el análisis del coleccionismo ligado al desarrollo institucional de los museos, enfoque adoptado en este trabajo. Para esto, ver entre otros trabajos Akin, 1996; Belk, 1995; Elsner y Cardinal, 1994; Pearce, 1992, 1994, 1997, 1998a, 1998b; Podgorny, 2000a, 2000b; Pomian, 1990; Sheets-Pyenson, 1988; Stocking, 1985; Walker, 1997.

2 Entre ellos el Museo Nacional de Buenos Aires, que dependía del Ministerio de Instrucción Pública y que hasta 1881 había sido el Museo Público de la provincia de Buenos Aires (éste creado en 1823); el Museo de Farmacología y Botánica de la Facultad de Ciencias Médicas, creado en 1900 y el Etnográfico de la Facultad de Filosofía y Letras, en 1906, ambos dependientes de la Universidad de Buenos Aires; los Museos Zoológico y Mineralógico y el Museo Antropológico y Paleontológico de la Universidad de Córdoba; el Museo de La Plata, creado bajo la administración del Ministerio de Obras Públicas de la provincia de Buenos Aires, que en 1906 con la creación de la Universidad de La Plata pasó a su jurisdicción; el Museo Histórico Nacional, creado en 1891 y el Museo Naval de la Policía Federal, en 1899. Entre los provinciales fueron creados el Museo Provincial de Paraná en Entre Ríos que cerró sus puertas en 1899 y el Museo del Colegio Nacional de Tucumán; el Museo Antropológico y Arqueológico de la provincia de Buenos Aires, creado en 1877 con la donación de la colección de Francisco Moreno, la cual pasaría a formar parte del Museo de la Plata (Lascano, 1980; Podgorny, 1993, 1995, 1997, 2000a, 2000b; Sauro, 2000; Sheets-Pyenson, 1988).

${ }^{3}$ Este concepto describe a los "museos pequeños e íntimos, situados generalmente lejos de las grandes ciudades y que a menudo tienen su origen en una sola persona" (Rosso, 1991, p. 242). Farró Fonalleras también considera que esta definición sirve para denominar a los museos que han nacido "a raíz de donaciones en los que los nombres de quienes los fundaron, en lugar de perderse en el anonimato, perviven en centros que conservan el carácter unitario que les infundió el fundador" (Farró Fonalleras, 1995, p. 54). Usamos esta caracterización ya que la naturaleza de estas instituciones no responde a las categorías señaladas por otros autores. Tal es el caso de los patrones que Pomian (1990) señala en la formación de museos públicos. El autor distingue cuatro modelos: el 'tradicional', representado por instituciones que han crecido a partir de colecciones y que estaban abiertos al público en contadas ocasiones; el 'revolucionario' que caracteriza a museos originados por decretos y controlados por el estado; el 'evergetic' cuyas colecciones fueron donadas por sus propietarios después de su muerte; y el 'comercial', formado a partir de la compra de colecciones (Pomian, 1990). En el caso de estudio, el origen de estos museos es similar en algunos aspectos al descripto por Lopes y Podgorny (m.s.) para el Museo de La Plata (Argentina) en el que el estado aceptó la donación de colecciónes al mismo tiempo que permitió que el control de las mismas estuviera bajo la supervisión de su donador.

4 Esto entraba en franca contradicción con la ley 9080 que a nivel nacional rige, en materia de patrimonio arqueológico, la ley 9080, sancionada en 1913 y reglamentada en 1921. Esta ley declaraba propiedad de la nación las ruinas y yacimientos arqueológicos y paleontológicos de interés científico y confería al Ministerio de Justicia e Instrucción Pública de la Nación la facultad de otorgar permisos (sólo a instituciones científicas nacionales o extranjeras) para utilizar o explotar dichos yacimientos con el asesoramiento de los museos Nacional de Historia Natural, Etnográfico de la Facultad de Filosofía y Letras de la Universidad de Buenos Aires 
y el Museo de La Plata (Berberián, 1992; Endere, 2000; Endere y Podgorny, 1997; Podgorny, 2000a). Se desprende claramente del texto que la acción de estos coleccionistas de provincia no estaba permitida de acuerdo a esta legislación.

${ }^{5}$ Esta percepción de sí mismo la expresa Luis Scalese al presentarse epistolarmente a un pariente italiano. Carta de Luis Scalese a Michele Gigliotti, 28 de julio de 1958. Archivo Administrativo Museo Histórico Regional Conrado Villegas de Trenque Lauquen (AMHTL).

${ }^{6}$ Contrato firmado el 27 de agosto de 1960, Letra H.D., Expediente 2395, 1960. Universidad Nacional del Sur.

7 Expediente $n^{\circ} 403$, letra S, 1956. Universidad Nacional del Sur.

8 Resolución Ministerial nº 3864/53. Ministerio de Educación de la Provincia de Buenos Aires.

${ }^{9}$ Disposición no 1 de la Dirección de Museos, Reservas e Investigaciones Culturales, 8 de julio de 1955.

10 El museo contó desde 1946 con el subsidio municipal de \$ $20 \mathrm{~m} / \mathrm{n}$ para barrido y limpieza de las salas del museo y gastos para compras generales. También obtuvo entre 1947 y 1951 un subsidio mensual de \$ 150 m/n otorgado por el Ministerio de Hacienda, Economía y Previsión de la provincia para ser usados en el pago del alquiler del local, \$ $100 \mathrm{~m} / \mathrm{n}$, y \$ $50 \mathrm{~m} / \mathrm{n}$ asignados a Luis Scalese como contribución por viajes, atención y cuidado de las colecciones (AMHTL).

${ }^{11}$ Los reclamos y lamentos se reiteran tanto a sus colegas como a los funcionarios. En carta del 2 de noviembre de 1952, Luis Scalese le escribía al Director de Museos Históricos de la Provincia, Dr. Juan G. Beltrán, para reclamarle una deuda que según su estimación era de $\$ 6.645,60$ ctvs. $\mathrm{m} / \mathrm{n}$ de enero a octubre de 1952 en concepto de alquiler, limpieza, luz y franqueo. Fundamentó este reclamo en el valor inmobiliario conferido a su propiedad con un tono terminal: "Excusará si en este día simbólico, elevo este S.O.S. a su digna consideración por si, eventualmente, con el Nuevo Presupuesto provincial podría contribuir con algo para solventar el estado económico de este Museo". Esta situación de ausencia de presupuesto provincial continuó aún retirado Scalese. El museo contaba sólo con un portero y la provincia le había hecho el ofrecimiento de trasladar a esa dependencia a una docente en tareas pasivas. Esta fue una propuesta que el Ministerio de Educación realizó en febrero de 1959 a los siguientes museos: cuatro para el Colonial e Histórico de Luján y uno por cada uno de los siguientes museos: Histórico Regional General Conrado Villegas de Trenque Lauquen; Parque Criollo y Museo Gauchesco de San Antonio de Areco; Histórico Regional Almirante Brown de Bernal (Quilmes); Museo y Parque Evocativo Los Libres del Sud de Dolores y Pampeano de Chascomús. En el caso de Trenque Lauquen no pudo aprovecharse esta resolución porque en la ciudad había una sola maestra en estas condiciones y no aceptó el ofrecimiento.

También en 1959 fue anunciada la construcción de un edificio para el Museo en el Parque Municipal con una inversión de la provincia de $\$ 1.300 .000 \mathrm{~m} / \mathrm{n}$ pero esto fue dilatándose y de este modo funcionó en la casa de Luis Scalese hasta el 6 de mayo de 1963. Luego de estar un tiempo cerrado fue trasladado a otro edificio.

12 Carta de Luis Scalese a Galileo J. Scaglia, Director del Museo Municipal de Ciencias Naturales y Tradicional de Mar del Plata, 10 de febrero de 1952.

13 Carta de Gabriel Campomar Cervera a Manuel Bejarano, Director de Museos, Monumentos e Investigaciones Culturales, Salliqueló, 4 de mayo de 1965. Archivo Administrativo Museo Histórico Regional Gabriel Campomar Cervera, Salliqueló (AMHS).

14 Carta de Scalese a Campomar Cervera del 2 de junio de 1959 en respuesta a una carta de Campomar solicitando que le informe sobre los trámites que debía hacer para ofrecer su colección a la provincia (AMHTL).

15 Expediente $n^{\circ}$ 259076/54. Dirección de Museos, Reservas e Investigaciones Culturales. Dirección General de Cultura. Ministerio de Educación. Trámite de expediente. Archivo Julio-Diciembre 1955. Archivo Dirección de Museos, Monumentos y Sitios Históricos, La Plata.

16 Estos consejos se derivaban de su propia experiencia. De este grupo de coleccionistas, Antonio Crespi Valls fue el único que contó con sueldo municipal por su función de director. Desde 1951 a 1953 obtuvo la categoría de Auxiliar 2 Director con un sueldo de $\$ 650 \mathrm{~m} / \mathrm{n}$ y a partir de ese año la categoría de Auxiliar 1 Director con los siguientes sueldos: 1953: \$ $90 \mathrm{~m} / \mathrm{n} ; 1954: \$ 1.000 \mathrm{~m} / \mathrm{n} ; 1955: \$ 1.100 \mathrm{~m} / \mathrm{n} ; 1957: \$ 1.400 \mathrm{~m} / \mathrm{n}$; enero de 1959 : $\$ 4.200 \mathrm{~m} / \mathrm{n}$. Expediente 86-M-1959, Municipalidad de Bahía Blanca. Por otro lado, el museo contó con presupuesto municipal dentro de la partida adjudicada a la Secretaría de Cultura. En el presupuesto del año 1951 los gastos de la Secretaría sumaban $\$ 120.000 \mathrm{~m} / \mathrm{n}$ y el museo contaba con $\$ 50.000 \mathrm{~m} / \mathrm{n}$. En el año 1959, con el mismo presupuesto asignado a la Secretaría de Cultura, el museo contó con $\$ 20.000 \mathrm{~m} / \mathrm{n}$ porque en el transcurrir de estos ocho años se fueron agregando más actividades a la gestión cultural, como la colonia de vacaciones, el Salón Regional de Arte de Bahía Blanca, actos culturales y fondos para actividades deportivas (Municipalidad de Bahía Blanca, 1952, 1960).

17 Carta de Antonio Crespi Valls a Emma Nozzi, 7 de noviembre de 1951. Archivo Administrativo Museo Histórico Regional Francisco de Viedma, Carmen de Patagones (AMHCdeP).

18 Carta de Luis Scalese a Emma Nozzi, 16 de febrero de 1952 (AMHTL). 
19 Carta de Gabriel Campomar Cervera a Antonio Crespi Valls, 24 de octubre de 1958, Archivo Museo y Archivo Histórico de Bahía Blanca (AMHBB).

20 Carta del Dr. Juan Beltrán, Director de Museos Históricos, a Luis Scalese, 21 de agosto de 1951. Nota 2091 (AMHTL).

21 Carta de Luis Scalese a Emma Nozzi, 16 de febrero de 1952 (AMHTL).

22 Carta de Luis Scalese a Antonio Crespi Valls, 4 de febrero de 1952 (AMHTL).

${ }^{23}$ Carta de Emma Nozzi a Luis Scalese, 9 de febrero de 1952 (AMHTL).

${ }^{24}$ Es importante la literatura que analiza las estrategias que han utilizado los museos metropolitanos del siglo XIX en Argentina para crear, mantener y acrecentar las colecciones de estas instituciones. Entre ellas se destacan los viajes de estudio y de exploración realizados por los museos (Podgorny, 1999b, 2000b); el establecimiento de redes internacionales con otras instituciones científicas a través de las que circulaban objetos, catálogos y publicaciones por medio de compra y/o donación e intercambio (Lopes, 1999, 2000; Lopes y Podgorny, 2000, 2001; Farro, 1999; Pérez Gollán, 1999; Podgorny, 1999a); y la obtención de redes que funcionaron en el contexto local y que vinculaban a las ciudades con el mundo rural, construyéndose como una estrategia para obtener materiales que estaban en lugares distantes de los centros científicos, tal como la red que estableció el Museo Etnográfico de Buenos Aires bajo la dirección de Juan Ambrosetti (1904-1917) entre esta institución, el ejército y los gobernadores provinciales (Pegoraro, m.s.).

25 Carta de Crespi Valls a Campomar Cervera, 5 de agosto de 1952 (AMHBB).

26 Pegoraro menciona la utilización de esta práctica para incorporar materiales a las colecciones del Museo Etnográfico. Esta institución enviaba a sus colaboradores rurales los criterios necesarios para la recolección de objetos, los que respondían a criterios cualitativos que incluían el diseño, decoración, técnicas de manufactura y procedencia y criterios cuantitativos, ya que los duplicados enviados servían para el canje con otros museos.

27 Carta de Crespi Valls a Campomar Cervera, diciembre de 1952 (AMHBB).

28 Para el 30 de diciembre de 1957, el inventario de bienes del museo de Trenque Lauquen era de 8.156 piezas con un valor simbólico de \$238.988 m/n, valor adjudicado por personal técnico de la Dirección de Museos, Reservas e Investigaciones Culturales. La casa de Luis Scalese tenía un valor de venta de \$250.000. Carta de Scalese a Eduardo Jonqueiras, Director del Departamento Técnico de la Unesco, 15 de julio de 1958 (AMHTL).

${ }^{29}$ Carta de Campomar Cervera a Crespi Valls, noviembre de 1952 (AMHBB).

30 Carta de Crespi Valls a Campomar Cervera, 1952 (AMHBB).

\section{REFERENCIAS BIBLIOGRÁFICAS}

Akin, Marjorie

1996

\section{Archivo Histórico \\ de la Provincia de \\ Buenos Aires}

1951

Arecco, Norberto 1955

Belk, Russel 1995

Berberián, Eduardo 1992

Bernard, Tomás Diego 1957

Bourdieu, Pierre 1997
Passionate possesion. The formation of private collections. In: Kingery, David (ed.). Learning from things. Method and theory of material culture studies. Londres, Smithsonian Institution Press, p. 102-28.

Primer Congreso de Historia de los pueblos de la provincia de Buenos Aires. La Plata, Dirección de Impresiones Oficiales.

Cinco años al frente del Departamento Ejecutivo 1 de Mayo de 1950-30 de Abril de 1955. Bahía Blanca, Municipalidad de Bahía Blanca.

Collecting in a consumer society.

Londres, Routledge.

La protección jurídica del patrimonio arqueológico en la República Argentina. Córdoba, Comechingonia.

Experiencias en museografía histórica.

Buenos Aires, Ediciones Anaconda.

Razones prácticas: sobre la teoría de la acción.

Barcelona, Editorial Anagrama. 
Dirección General del Servicio Estadístico

Nacional 1951

Elsner, John y

Cardinal, Roger 1994

Endere, María Luz 2000

Endere, María Luz y Podgorny, Irina 1997

Farro, Máximo 1999

Farró Fonalleras, Dolores 1995

Kopytoff, Igor 1991

Lascano, González A. 1980

Lopes, Maria Margaret 2000

Lopes, Maria Margaret 1999

Lopes, Maria Margaret y Podgorny, Irina 2001

Lopes, Maria Margaret y Podgorny, Irina 2000

Lopes, Maria Margaret y Podgorny, Irina

Ministerio de Educación 1979

Ministerio de Gobierno 1954

Municipalidad de Bahía Blanca 1960

Municipalidad de Bahía Blanca 1952

Municipalidad de Bahía Blanca 1951
Censo de 1947. IV Censo General de Población; resultados generales. Buenos Aires.

The cultures of collecting. Cambridge, Harvard University Press.

Arqueología y legislación en Argentina. Cómo proteger el patrimonio arqueológico. Buenos Aires, Universidad Nacional del Centro de la Provincia de Buenos Aires.

Los gliptodontes son argentinos: la ley 9080 y la creación del patrimonio nacional. Ciencia Hoy, v. 7, n. 12, p. 54-9.

The business of museums. Towards a history of collections in La Plata's Museum, 1888-1906. World Archaeological Bulletin, n. 10, p. 13-21.

Los coleccionistas y sus museos. Hacia una tipología específica. Museum, v. 185, n. 1, p. 54-8.

La biografía cultural de las cosas: la mercantilización como proceso. In: Appadurai, Arjun (ed.). La vida social de las cosas. Perspectiva cultural de las mercancías. México, Grijalbo, p. 89-122.

El Museo de Ciencias Naturales de Buenos Aires. Su historia. Buenos Aires, Ediciones Culturales Argentinas.

Nobles rivales: estudios comparados entre el Museo Nacional de Río de Janeiro y el Museo Público de Buenos Aires. In: Montserrat, Marcelo (org.). La ciencia en la Argentina entre siglos. Textos, contextos e instituciones. Buenos Aires, Manantial, p. 277-96.

Sociedades científicas e museus na América Latina no século XIX. Saber y Tiempo 7, v. 2, p. 51-72.

The shaping of Latin American museums of natural history, 1850-1990. Osiris, p. 108-18.

Caminos cruzados: el Museo Nacional de Historia Natural de Montevideo en la documentación del Museo Nacional de Buenos Aires. Ciencia Hoy, v. 10, n. 57, p. 42-50.

El desierto en una vitrina. Los museos de ciencias en la Argentina. (mimeo.)

Catálogo de museos, monumentos y lugares históricos de la provincia de Buenos Aires. La Plata, Ministerio de Educación.

Congreso Extraordinario de Municipios de la Provincia. La Plata, Ministerio de Gobierno de la Provincia de Buenos Aires.

Memoria y balance financiero. Ejercicio 1959. Boletín Municipal, n. 412, año XXXVIII, p. 15.522-66.

Memoria y balance financiero. Ejercicio 1951.

Boletín Municipal, n. 361-6, XXXI, p. 12.204-13.125.

Museo Histórico Municipal. Boletín Municipal de Bahía Blanca, n. 349-54, XXX, p. 12.084. 
Municipalidad de Bahía Blanca 1943

Noticias. 1952

Pearce, Susan 1998a.

Pearce, Susan $1998 b$.

Pearce, Susan 1997

Pearce, Susan 1995

Pearce, Susan 1994

Pearce, Susan 1992

Pegoraro, Andrea

Pérez Gollán, José 1999

Podgorny, Irina 2000a

Podgorny, Irina 2000b

Podgorny, Irina 1999a

Podgorny, Irina 1999b

Podgorny, Irina 1997

Podgorny, Irina 1995

Podgorny, Irina 1993

Pomian, Krysztof 1990

Rosso, Aroldo 1991

Sanguinetti de Bórmida, Amalia 1963
Creación del Museo y Archivo Histórico Municipal.

Boletín Municipal de Bahía Blanca, n. 255-6, XXII, p. 9.521-2.

20 de junio, Salliquelló.

Interpreting objects and collections.

Londres, Routledge.

Collecting in contemporary practice.

Londres, Sage Publications.

Experiencing material culture in the Western World.

Leicester, University Press.

On collecting: an investigation into collecting in the European tradition (collecting cultures). Londres, Routledge.

Interpreting objects and collections.

Londres, Routledge.

Museums, objects, and collections.

Washington, Smithsonian Institution Press.

Estrategias de formación de colecciones del Museo Etnográfico. 1904-1917. Funcionarios de gobierno en la recolección de piezas. (mimeo.)

Mr. Ward en Buenos Aires. Los museos y el proyecto de nación a fines del siglo XIX. Ciencia Hoy, v. 5, n. 28, p. 52-8.

El argentino despertar de las faunas y las gentes prehistóricas.

Buenos Aires, Eudeba.

Los gliptodontes en París: la colección de mamíferos fósiles pampeanos en los museos europeos del siglo XIX. In: Montserrat, Marcelo (org.).

La ciencia en la Argentina entre siglos. Textos, contextos e instituciones.

Buenos Aires, Manantial, p. 309-29.

Desde la tierra donde los monstruos aún no tienen nombre.

El ordenamiento de la naturaleza a través de los museos y de la ciencia en la Confederación Argentina. Quipu, v. 12, n. 2, p. 167-86.

La patagonia como santuario natural de la ciencia finisecular. Redes, v. VI, n. 14, p. 157-76.

El museo soy yo: Alfred Marbais Du Graty en la Confederación Argentina. Ciencia Hoy, p. 48-53.

De razón a facultad: ideas acerca de las funciones del Museo de La Plata en el período 1890-1918. Runa, 22, p. 89-104.

Arqueología y educación. La inclusión de la arqueología pampeana en la educación argentina. Tesis de Doctorado, Universidad Nacional de La Plata, La Plata.

Collectors $\&$ curiosities: Paris and Venice, 1500-1800.

Londres, Polity Press.

En Argentina, un museo de padre único.

Museum, v. 172, n. 4, p. 242-4.

La preparación de museólogos en la Universidad Nacional de Buenos Aires. Boletín de la Dirección de Museos, n. II, p. 13-5. 
Sauro, Sandra

2000

Sheets-Pyenson, Susan 1988

Silva Montaner, Raúl $1959 a$

Silva Montaner, Raúl $1959 b$

Stocking, George 1985

Walker, Julian 1997
El Museo Bernardino Rivadavia, institución fundante de las ciencias naturales en la Argentina del siglo XIX. In: Montserrat, Marcelo (org.). La ciencia en la Argentina entre siglos. Textos, contextos e instituciones. Buenos Aires, Manantial, p. 329-44.

Cathedrals of science: the development of colonial natural history museums in the late nineteenth century. Kingston, McGill-Queens.

La primera escuela de museología del país.

Boletín de la Dirección de Museos, II, p. 13-7.

La escuela de museología del Museo Social Argentino.

Boletín del Museo Social Argentino, v. XXXVII, n. 309, p. 17- 20.

Objects and others: essays on museums and material culture. In: Stocking, George (ed.). History of anthropology, v. 3. Madison, University of Wisconsin Press.

Afterword: acquisition, envy and the museum visitor. In: Pearce, Susan (ed.). Experiencing material culture in the Western World. Leicester, Leicester University Press.

Recebido para publicação em janeiro de 2003.

Aprovado para publicação em outubro de 2004. 